\title{
Análisis de la asignación presupuestaria a las universidades nacionales paraguayas en el período del Presidente Horacio Cartes
}

\author{
Ever F. Villalba Benítez \\ evervill@gmail.com \\ Rolando Ortega \\ rolando4224@gmail.com \\ Investigadores \\ Pronii Conacyt / \\ FCTA Universidad Nacional de Pilar
}

\section{RESUMEN}

El desafío de las instituciones de educación superior en el último quinquenio, no es conseguir mayor presupuesto, sino el de mantenerlo. En este artículo se analiza la asignación presupuestaria a las universidades públicas, en el periodo de gobierno del Presidente Horacio Cartes (2013-2018) comparando con la inflación registrada. La investigación adopta un enfoque cuantitativo, toma datos del Ministerio de Hacienda y el Banco Central del Paraguay; es de diseño observacional, longitudinal, retrospectivo y descriptivo. La inversión en las universidades decreció en un 3,35\% teniendo en cuenta el porcentaje de inflación acumulada, por ende tampoco llegó al 7\% del presupuesto destinada a la Administración Central, dejando en evidencia que las acciones del gobierno no tiene correlación con las cuestiones declarativas y legales, en cuanto a la educación superior como bien público y un derecho humano.

Palabras claves: Universidad, Enseñanza Pública, Financiación de la Educación, Bien Público. 


\title{
Analysis of the budget allocation to Paraguayan national universities in the period of President Horacio Cartes
}

\begin{abstract}
ABSTRAC
The challenge of higher education institutions in the last five years is not to get a bigger budget, but to maintain it. This article analyzes the budgetary allocation to public universities, during the term of President Horacio Cartes (2013-2018), comparing it with the inflation registered. The research takes a quantitative approach, taking data from the Ministry of Finance and the Central Bank of Paraguay; It is observational, longitudinal, retrospective and descriptive in design. Investment in universities decreased by $3.35 \%$ taking into account the percentage of accumulated inflation, therefore it also did not reach $7 \%$ of the budget allocated to the Central Administration, making it clear that government actions have no correlation with issues declarative and legal, regarding higher education as a public good and a human right.
\end{abstract}

Key words: University, Public Education, Financing of Education, Public Good.

Artículo recibido: 17. marzo. 2020 Aceptado para publicación: 11. junio. 2020 Correspondencia: rolando4224@gmail.com Conflictos de Interés: Ninguna que declarar 


\section{INTRODUCCIÓN}

Uno de los grandes desafíos de las universidades de América Latina para este nuevo siglo sigue siendo el financiamiento, para Misas Arango (2011) cada vez las instituciones de educación superior necesitan más presupuesto para cumplir con sus funciones sustantivas y el estado, principal financiador, no está dispuesto a conceder sin contrapartida. La exigencia de contrapartidas para mejorar el presupuesto se fundamenta, en los lineamientos de la aplicación de políticas públicas del Gobierno de Cartes administración pública, cuya praxis en la gestión del estado se ajusta a la lógica del liberalismo económico, que en muchos casos distan o son las antípodas del quehacer universitario.

Es evidente que, tanto en el país como en la región, se tienen posturas antagónicas, por un lado, los que pregonan la educación superior como un bien público, y por otro, los que quieren someterla a las reglas de mercado considerándola como un servicio comercializable; si bien en Paraguay la educación superior está arancelada, en países como Argentina y Brasil es gratuita. La intención de organismos internacionales como el Banco Mundial son claras y apuntan a favorecer a la privatización de los estudios universitarios, en el año 2012 recomienda al Brasil, que es necesario introducir el arancelamiento de la educación superior porque no existe correlación con el aumento de la matrícula en las instituciones públicas (Banco Mundial, 2017).

La Declaración Universal de los Derechos humanos establece, sin hacer diferencias en los subsistemas, que todas las personas tienen derecho a la educación En relación a la educación universitaria refiere, que el acceso será para todos de acuerdo a los méritos (Asamblea General de la ONU, 1948).

En el mismo sentido la constitución del Paraguay establece sin distinción de niveles, que toda persona tiene derecho a la educación permanente y completa, en cuanto a la educación superior dice: "El Estado fomentará la enseñanza media, técnica, agropecuaria, industrial y la superior o universitaria, así como la investigación científica y tecnológica" (Constitución Nacional de la República del Paraguay, 1992).

En la Conferencia Regional de Educación Superior realizada en Cartagena de Indias en el año 2008, que reunió a académicos y representantes de países latinoamericanos y 
caribeños, Paraguay uno de esos países, declaró que la "educación superior es un bien público social, un derecho humano y universal y deber del Estado", (CRES, 2008). El siguiente año, 2009, se realiza en París la Conferencia Mundial sobre la Educación Superior, al cual asistió la representación de Paraguay, donde se consensuó la declaración que señala a "la educación superior en tanto que bien público, es responsabilidad de todas las partes interesadas, en particular de los gobiernos" (UNESCO, 2009).

Es evidente que la postura latinoamericana y caribeña fue disminuida en el acuerdo a que se llegó en la conferencia mundial, si bien se mantuvo en el comunicado en el carácter de la educación como bien público, no lo declara como social, menos derecho humano fundamental universal y responsabilidad del estado. En cuanto al financiamiento, en el comunicado de la conferencia del 2009, propone la estimulación de la inversión privada, pues reconoce que los fondos públicos son limitados y debe buscar otras formas de financiar, sugiriendo como alternativa la inversión público-privada (UNESCO, 2009).

Paraguay recoge los postulados de la declaración de la conferencia regional del año 2008 y la establece como ley, así como se puede observar en la actual ley de educación superior sancionada en el año 2013. La ley de educación superior, en consonancia con lo que establece la constitución de la República del Paraguay, eleva a la educación superior como un bien público y como un factor principal para desarrollar el país, en un sistema democrático y equitativo; asimismo, reconoce como un derecho humano fundamental para los que estén en condiciones legales y académicas para gozarla, por lo que es responsabilidad del estado la organización, administración, dirección y gestión de dicho sistema (Ley N 4995, 2013).

El aporte del estado es la principal fuente de financiación del sistema educativo del Paraguay y está establecido anualmente los recursos asignados para cada universidad pública en el Presupuesto General de Gastos de la Nación (OEI, 1992). En la carta magna se establece que los recursos destinados a educación no debe ser inferior al $20 \%$ del total asignado a la Administración Central (Constitución Nacional de la República del Paraguay, 1992), por otra parte la UNESCO recomienda que la inversión en educación no sea menor al 7\% del PIB (Palacios, 2009). La reciente ley de educación superior establece que la inversión en educación superior no debe ser menor al 7\% de lo destinado a la Administración Central excluido los préstamos (Ley No 4995, 2013). 
Las universidades paraguayas gozan de autonomía de acuerdo a Constitución Nacional de la República del Paraguay, (1992) y es reglamentada en la Ley de Educación Superior, en la misma normativa se establece que las públicas cuentan con "autarquía financiera para generar, administrar y disponer de sus fondos, correspondientes al Presupuesto General de la Nación" (Ley No 4995, 2013).

Para Rinesi, la educación superior como bien público y un derecho humano fundamental prácticamente es una consigna de los referentes académicos, en especial en el sector público, en las diferentes declaraciones y comunicados de los encuentros regionales y mundiales sobre educación superior, pero no vale la pena emocionarse porque solo se trata de eso, una declaración y no se traduce en lo ejecutivo (2016). En el Paraguay estas declaraciones son recogidas en el derecho positivo y entró en vigencia un mes antes del período de gobierno del Presidente Horacio Cartes, por lo que esta investigación analiza la inversión estatal durante su mandato y compara con el índice de inflación.

En el marco del desarrollo de esta línea de investigación los autores han publicado otros informes como los titulados Financiamiento de la educación superior en Paraguay: inversión estatal en Universidades Públicas entre el 2007 al 2018 (2018) y Periodos presidenciales y la inversión estatal en Universidades Públicas Paraguayas (2019).

\section{Objetivo General}

Analizar la evolución de la asignación presupuestaria a las universidades nacionales en el periodo de gobierno del presidente Horacio Cartes, comparando con la inflación por año y acumulada.

\section{METODOLOGÍA}

El trabajo adopta un enfoque cuantitativo (Sautu, Boniolo, Dalle, \& Elbert, 2005), con diseño observacional, retrospectivo, longitudinal, y descriptivo (Supo, 2012). No se manipulan variables en el proceso, los datos son recogidos de fuentes secundarias de datos públicos del Ministerio de Hacienda y el Banco Central del Paraguay del periodo estudiado 2013 al 2018, con el análisis se pretende caracterizar el fenómeno estudiado (Salkind, 1999). 
Los presupuestos estudiados corresponden a los aprobados durante el gobierno del presidente Horacio Cartes, los del 2014 al 2018, además se incorpora para referencia y punto de partida el correspondiente al ejercicio 2013. En cuanto a la inflación se parte del acumulado al cierre del año 2013 y los sucesivos hasta el 2017, se tomaron estos últimos considerando que los presupuestos a ser aprobados para el siguiente ejercicio deberían cubrir por lo menos la tasa de inflación del ejercicio fenecido.

\section{RESULTADOS Y DISCUSIÓN}

Paraguay es uno de los últimos países en contar con universidad, la primera en crearse es la Universidad Nacional de Asunción en el año 1889, 350 años después de la Universidad de Santo Domingo, que se fundó en el año 1538 y considerada la más antigua de Latinoamérica (Carbone \& Giménez, 2015).

Por varios años la Nacional de Asunción fue la única universidad del país, recién en el año 1960 se crea una segunda de gestión privada y es la Católica de Asunción, estas dos instituciones lideran y monopolizan la educación superior por varios años, mediante el advenimiento de la democracia en 1989, se vive un proceso de libertad y da posibilidad a la creación de nuevas universidades creándose hasta la fecha 55, de las cuales 9 son de gestión pública y 46 de gestión privadas.

\section{Inversión estatal en las universidades públicas del Paraguay}

En la siguiente tabla se observa la composición de las universidades públicas paraguayas distribuidas en base a las leyes de creación y los presupuestos que se les asignaron años tras años y en el periodo de gobierno de Horacio Cartes. 
Tabla 1 Presupuesto de Universidades Nacionales en dólares (1\$ = 5.900 Gs.)

\begin{tabular}{|c|c|c|c|c|c|c|c|}
\hline Universidades & 2014 & 2015 & 2016 & 2017 & 2018 & Acumulado & $\%$ \\
\hline Asunción (1889) & 206.883 .533 & 216.769 .801 & 211.866 .966 & 218.285 .598 & 226.405 .491 & 1.080 .211 .389 & 76,86 \\
\hline Este (1893) & 18.010 .826 & 18.618 .634 & 18.587 .597 & 18.917.532 & 19.093.299 & 93.227 .889 & 6,63 \\
\hline Pilar (1994)* & 7.806 .941 & 8.113 .515 & 8.220 .315 & 8.220 .315 & 8.780 .941 & 41.142 .028 & 2,93 \\
\hline Itapúa (1996) & 9.388 .393 & 10.217 .356 & 10.955 .872 & 11.358 .293 & 10.874 .076 & 52.793 .990 & 3,76 \\
\hline Concepción (2007) & 6.026 .438 & 6.889 .311 & 7.276 .561 & 7.344 .351 & 7.330 .222 & 34.866 .884 & 2,48 \\
\hline Villarrica (2007) & 7.692 .363 & 8.039 .149 & 8.137 .803 & 8.137 .803 & 8.769 .975 & 40.777 .093 & 2,90 \\
\hline Caaguazú (2007) & 8.084 .579 & 8.766 .193 & 9.066 .615 & 9.066 .615 & 9.347 .135 & 44.331 .137 & 3,15 \\
\hline Canindeyú (2010) & 2.829 .541 & 3.426 .278 & 3.884 .461 & 3.961 .434 & 4.064 .800 & 18.166 .515 & 1,29 \\
\hline Py - Taiwán (2018) & 0 & 0 & 0 & 0 & 0 & 0 & 0 \\
\hline Total & 266.722 .613 & 280.840 .238 & 277.996 .191 & 285.291 .943 & 294.665 .939 & 1.405.516.924 & 100,00 \\
\hline
\end{tabular}

Fuente: Elaboración propia a partir de datos del Ministerio de Hacienda y ANEAES

Lo que recibe la universidad más antigua, es tres veces más de lo que reciben las demás universidades en su conjunto, esto representa el 76,86\% del total asignado para las universidades nacionales; esta situación se arrastra de épocas anteriores (Villalba Benítez, Educación superior en Paraguay: análisis de la inversión estatal en Universidades Públicas entre el 2007 al 2016, 2017), pero tampoco en el periodos analizado se visualizaron acciones para disminuir la brecha.

En la época analizada se crea la última universidad pública, Paraguay - Taiwán a espaldas del Consejo Nacional de Educación Superior, que según la Ley de Educación Superior, debe dictaminar sobre la creación de una institución superior y dicha opinión es vinculante según la Ley Educación Superior. (Ley No 4995, 2013).

Como se menciona más arriba la Ley de Educación Superior establece que el presupuesto destinado a la Educación Superior no debe ser inferior al 7\% de lo destinado a la Administración Central, seguidamente se presenta una tabla para verificar el cumplimiento de la norma. 
Análisis de la asignación presupuestaria...

Tabla 2 El 7\% de la Administración Central y lo asignado a las universidades

\begin{tabular}{lrrrrr}
\hline Presupuestos & \multicolumn{2}{l}{$\mathbf{2 0 1 4}$} & \multicolumn{2}{l}{$\mathbf{2 0 1 5}$} & \multicolumn{2}{l}{$\mathbf{2 0 1 6}$} & \multicolumn{2}{l}{$\mathbf{2 0 1 7}$} & \multicolumn{2}{l}{$\mathbf{2 0 1 8}$} \\
\hline Adm. Central & 5.048 .289 .380 & 5.933 .577 .585 & 5.913 .982 .168 & 5.913 .982 .168 & 6.616 .585 .087 \\
7\% según Ley de ES & 353.380 .257 & 415.350 .431 & 413.978 .752 & 413.978 .752 & 463.160 .956 \\
Universidades & 251.967 .938 & 280.840 .238 & 277.996 .191 & 285.291 .943 & 294.665 .939 \\
Diferencia & -101.412 .319 & -134.510 .192 & -135.982 .561 & -128.686 .809 & -168.495 .017 \\
\% del Presp / AC & $4,99 \%$ & $4,73 \%$ & $4,70 \%$ & $4,82 \%$ & $4,45 \%$
\end{tabular}

Fuente: Elaboración propia.

Según los datos contenidos en la tabla, el presupuesto de las universidades nacionales llega apenas alrededor del 5\% de la Administración Central, que de acuerdo a la Ley de Educación Superior como mínimo debe ser del 7\% lo que demuestra un déficit del $2 \%$.

El presupuesto destinado a las universidades públicas paraguayas en el año 2013 ascendía a 251.967 .938 \$, se toma como base para calcular la variación interanual del presupuesto en el periodo estudiado, en el caso de la inflación se tiene en cuenta el índice al cierre del ejercicio anterior, el \% de inflación al 2013 es del 3,70 que sería el mínimo de aumento que debe considerarse para el presupuesto 2014 en este caso para dicho año aumentó $5,86 \%$, en la siguiente tabla observa el comportamiento de la inflación y los porcentajes de aumentos de presupuesto en el período analizado.

Tabla 2 Comparativo del aumento de presupuesto con la tasa de inflación

\begin{tabular}{lccccc}
\hline Descripción & $\mathbf{2 0 1 4}$ & $\mathbf{2 0 1 5}$ & $\mathbf{2 0 1 6}$ & $\mathbf{2 0 1 7}$ & $\mathbf{2 0 1 8}$ \\
\hline \% Inter-anual Presupuesto & 5,86 & 5,29 & $-1,01$ & 2,62 & 3,29 \\
Inflación & 3,70 & 4,20 & 3,10 & 3,90 & 4,50 \\
Diferencia (\% P - Inflación) & $\mathbf{2 , 1 6}$ & $\mathbf{1 , 0 9}$ & $\mathbf{- 4 , 1 1}$ & $\mathbf{- 1 , 2 8}$ & $\mathbf{- 1 , 2 1}$ \\
Acumulado \% Presupuesto & $\mathbf{5 , 8 6}$ & 11,15 & 10,14 & 12,76 & 16,05 \\
Acumulado Inflación & 3,70 & 7,90 & 11,00 & 14,90 & 19,40 \\
Diferencia (\% Acum P. - Inflación) & $\mathbf{2 , 1 6}$ & $\mathbf{3 , 2 5}$ & $\mathbf{- 0 , 8 6}$ & $\mathbf{- 2 , 1 4}$ & $\mathbf{- 3 , 3 5}$
\end{tabular}

Fuente: Elaboración propia a partir de los datos del BCP y el MH 
Como se puede observar en la tabla apenas el 16\% aumentó, en todo el período el presupuesto asignado a las universidades nacionales, en cambio la inflación acumulada fue mayor llegando al 19,40\%, lo que representa un déficit del 3,35\%.

Seguidamente un gráfico donde se compara el \% del aumento presupuestario y la inflación en el periodo analizado.

\section{Grafico 1 Comparación de la inflación con el \% de aumento presupuestario.}

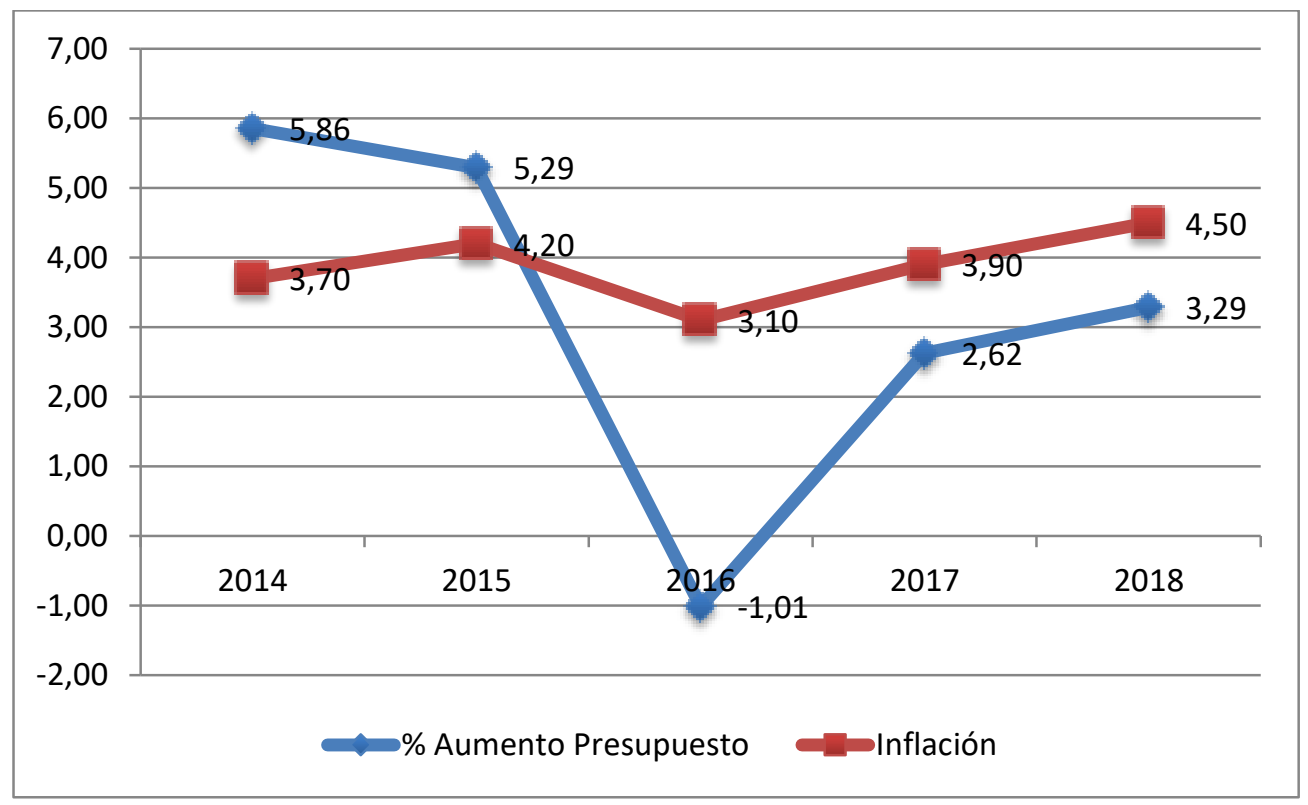

Fuente: Elaboración propia

Apenas los primeros dos años de gobierno del Presidente Horacio Cartes pudo equiparar el aumento del presupuesto de las universidades públicas con la inflación del año anterior, en el tercer año no solo no pudo compensar la inflación, sino que la asignación nominal, fue inferior al del año anterior y los últimos dos años; los pequeños aumentos son insuficientes para cubrir la inflación.

\section{CONSIDERACIONES FINALES.}

En el trabajo se analizó la asignación presupuestaria a las universidades públicas del Paraguay en el período de mandato del Presidente Horacio Cartes, permitió demostrar que las acciones del gobierno, no tienen correlación con las cuestiones declarativas y legales en cuanto al financiamiento de la educación superior; teniendo en cuenta lo que establece la Carta Magna, la Ley 4995/13 y las Declaraciones Regionales y Mundiales, 
del cual Paraguay es subscribiente, donde se establece que la Educación Superior es un Bien Público, un Derecho Humano Fundamental y Deber de los Estados.

Durante todo el período estudiado, el presupuesto a las Universidades Nacionales apenas llegó al 5\% de lo destinado a la Administración Central, que según la normativa vigente sobre Educación Superior debe ser al menos del 7\%, por otro lado teniendo en cuenta las devaluaciones que genera la inflación el total de lo asignado a las universidades durante la época de Cartes decreció en un 3,35\%.

Según el informe de Latinobarómetro del año 2018, la preocupación sobre los problemas de la educación de la población paraguaya ocupa el quinto lugar, con apenas 9\%, mientras que los temas que más preocupan son el desempleo con $29 \%$ y corrupción con $13 \%$, (Corporación Latinobarómetro, 2018), en el año 2013 la inquietud sobre el tema era incluso menor, apenas el 6,2\% (Corporación Latinobarómetro, 2013); es probable que los decisores sobre la distribución del presupuesto público hayan manejado este tipo de informaciones sobre la opinión de la ciudadanía, por lo que no asignaron mayor partidas presupuestarias a las instituciones de educación superior. 


\section{LISTA DE REFERENCIAS}

Albach, P., \& Knight, J. (2005). Vision Panoràmica de la Internacionalizacion de la Educacion Superior.

Ameneiros, J. (1998). Gestión de desechos sólidos urbanos en la Ciudad de la Habana. La Habana-Cuba.: Monografía ISPJAE.

Asamblea General de la ONU. (1948). Declaración Universal de los Derechos Humanos. Paris: Organización de las Naciones Unidad.

Baig, S., Courant, P., \& Liechti, P. (1999). Treatment of Landfill Leachates:Lapeyrouse and Satrod case studies. Ozone Science and Engineering.

Banco Mundial. (2017). Un ajuste justo: propuesta para aumentar eficiencia y equidad del gasto público en el Brasíl . Washington: Banco Mundial.

Carbone, R., \& Giménez, F. (2015). (Re)vuelta a la Universidad ¿Institución Perdida o Nunca Concretada? Publicación Digital del Centro de Estudios y Educación Popular Germinal , 1-41.

Cegarra, J. (2004). Residuos Sanitarios.Manual Ambiental. Lima.Peru.: Instituto Ambiental Peruano.

Cepis/Ops/Oms. (2003). Guía para el diseño,construcción y operación de rellenos sanitarios manuales. Capítulo 2. Santiago.Chile.

Constitución Nacional de la República del Paraguay (Convención Nacional Cosntituyente 20 de Junio de 1992).

Corporación Latinobarómetro. (2013). Opinión Pública Latinoamericana. Santiago de Chile: Corporación Latinobarómetro.

Corporación Latinobarómetro. (2018). Informe de 2018. Santiago de Chile: Corporción Latinobarómetro.

CRES. (2008). Declaración y Plan de Acción de la Conferencia Regional de la Educación Superior en América Latina y el Caribe. Cartagena de Indias: IESAL - UNESCO.

Ehrig, H. (1989). Leachate from Household Waste Landfills, the Quantity and Composition. Berlin.: Waste Handbook (Loose Leaf Collection).

Ehrig, H., Brinkman, U., Hôring, K., Heim, M., \& Hagedom, S. (1995). The Influence the Pre.treatment of Waste Behaviour of Emissions of Landfills as well as the Assessment by biological Tests. Wuppertal.U.S.A.: Department for Waste and Household Water Management.Bergische University. 
EPA. (1990). Waste minimization.EPA/530-SW-90-044. From http://www.epa.gov/

EPA. (2002). Enviromental Protection Agency.Office of Solid Waste.OSW 486.E.U. From http://epa.gov/epaoser/hazwaste/test/

Garatte, L., \& Casajùs, R. (2016). La movilidad Acadèmica como polìtica de intenacionalizaciòn.

Indian Resource Center. (2006). Table of Health Effects of Toxic Chemicals in Landfill Leachates. From http://www.indiaresaource.org/issues/water

Ley N ${ }^{o}$ 4995. (2013). De Educación Superior. Asunción: Congreso Nacional del Paraguay.

López, M., Espinosa, M., Ramos, C., Mayari, R., Pellón, A., \& Martinez, V. (2003). Desarrollo y tendencias en la gestión de residuos sólidos urbanos. La Habana.Cuba.: CNIC.

Maroto Vargas, \& Segura Arias. (1999). Estrategia de capacitación para el manejo de los desechos sólidos domiciliares. San José.Costa Rica.

Misas Arango, G. (2011). La financiación de la educación superior en América Latina: un análisis de caso 1990 - 2008. Análisi Político , 61-81.

Montes, V. (2004). Relleno Sanitario para RSD. San José.Costa Rica: Guia de Consultas Técnicas.IMBIO.

OEI. (1992). El financiamiento de la Educación . In OEI, Gasto Público en Educación. Asunción.

Oregioni, M., \& Piñero, F. J. (2012). Redes de producción y difusión de conocimiento: ¿Un instrumento para orientar la internacionalización de la Universidad Argentina hacia Latinoamérica?

Palacios, G. (2009). Financiación de la educación, sus logros y desafíos en el Paraguay. Población y Desarrollo , 39-51.

Rinesi, E. (2016). Filosofía y política de la Universidad. Buenos Aires: Ediciones UNGS.

Robinson., H. (1995). A Review of the Composition of Leachates from Domestic Wastes in Landfill Sites. London.UK.: UK Department of the Environment.Reference DE0918/FR1.

Salkind, N. J. (1999). Métodos de Investigación. México: Prentice Hall.

Sautu, R., Boniolo, P., Dalle, P., \& Elbert, R. (2005). Manual de metodología. Construcción del marco teórico, formulación de objetivos y elección de la 
metodología. Buenos Aires: Consejo Latinoamericano Ciencias Sociales CLACSO.

Supo, J. (2012). Seminarios de Investigación Científica. Metodología de la investigación científica para las ciencias de la salud. Arequipa: Bioestadístico.

Sutton, M. H., \& Canales Sanchez. (2016). Porque Moverse?

UNESCO. (2009). Comunicado Conferencia Mundial sobre la Educación Superior. París: UNESCO.

Villalba Benítez, E. F. (2017). Educación superior en Paraguay: análisis de la inversión estatal en Universidades Públicas entre el 2007 al 2016. Montevideo: https://www.cse.udelar.edu.uy/wp-content/uploads/sites/5/2018/02/CO-7Villalba.pdf.

Villalba Benítez, E. F., \& Ortega, R. (2018). Financiamiento de la educación superior en Paraguay: inversión estatal en Universidades Públicas entre el 2007 al 2018. Paraguay desde las Ciencias Sociales, 1-14.

Villalba Benítez, E. F., \& Ortega, R. (2019). Periodos presidenciales y la inversión estatal en Universidades Públicas Paraguayas. Brazilian Journal of Development, 23202332.

Obtenido

de http://www.brazilianjournals.com/index.php/BRJD/article/viewFile/1276/1148

Yugueros Robledo, M. (2015). La Educaciòn Superior en el Sector Educativo del Mercosur. 